\title{
CAPACIDAD DE ADSORCIÓN DE PLOMO CON INTERCAMBIADORES CATIÓNICOS DE CARBON XANTADO
}

\section{ADSORPTION CAPACITY OF LEAD WITH CATIONICS EXCHANGERS OF XANTED COAL}

\author{
Fredy Colpas Castillo ${ }^{1}$, Arnulfo Tarón Dunoyer ${ }^{2}$, Yelitza Vasquez ${ }^{3}$
}

\begin{abstract}
${ }^{1}$ Ingeniero Químico, M.Sc., Profesor investigador, Facultad de Ciencias Exactas y Naturales, Departamento de Química, Grupo de investigación Carboquímica. Universidad de Cartagena, Campus de San Pablo, Cartagena-Bolívar, Colombia, email: fcolpasc1@unicartagena.edu.co; ${ }^{2}$ Quimico Farmacéutico, M.Sc., Profesor investigador, Facultad de Ingenierías, Grupo de GIBAE. Universidad de Cartagena, Av. del Consulado Calle 30 \# 48-152, Cartagena-Bolívar, Colombia, e-mail: atarond@ unicartagena.edu.co; ${ }^{3}$ Quimico Puro, Facultad de Ciencias Exactas y Naturales, Departamento de Química, Grupo de investigación Carboquímica. Universidad de Cartagena, e-mail: yelitzavasquez2792@gmail.com
\end{abstract}

Rev. U.D.C.A Act. \& Div. Cient. 20(2): 425-433, Julio-Diciembre 2017

\section{RESUMEN}

En este estudio, se describe la adsorción de plomo $\left(\mathrm{Pb}^{2+}\right)$ en soluciones acuosas, a partir de carbón modificado, mediante tratamiento con $\mathrm{CS}_{2}$ y $\mathrm{NaOH}$, empleando una relación 16,6:1 NaOH/Carbón p/p y 4,08:1 CS2/carbón p/p. Se realizaron pruebas de $\mathrm{pH}$, cinéticas e isotermas de adsorción, para evaluar la capacidad de adsorción del carbón xantado y carbón no xantado. Los grupos azufre en el carbón xantado fueron identificados por espectroscopia infrarroja de reflectancia difusa (FTIR) y comparados con los espectros del carbón no xantado. Los resultados muestran que la mejor remoción de plomo en la solución con una concentración de $100 \mathrm{mg} \mathrm{de} \mathrm{Pb}^{2+} / \mathrm{L}$ y dosis de $50 \mathrm{mg}$ de adsorbente $/ 10 \mathrm{~mL}$, respectivamente, fue a un valor de $\mathrm{pH} 5,0$, logrando un porcentaje de adsorción de 98\%. Los estudios cinéticos indican que el proceso de adsorción puede alcanzar el equilibrio alrededor de 120min, siguiendo una cinética de pseudo-segundo orden, para ambos materiales. El estudio de las isotermas indica que el carbón se ajusta mejor a una isoterma de tipo Langmuir y cuando se somete a la xantación, se ajusta a una isoterma de tipo Freundlich.

Palabras clave: Carbón, isoterma Langmuir, xantatos, efluentes industriales.

\section{SUMMARY}

This study describes the adsorption of lead $\left(\mathrm{Pb}^{2+}\right)$ in aqueous solutions of modified coal by treatment with $\mathrm{CS}_{2}$ and $\mathrm{NaOH}$, using a ratio of 16,6:1 NaOH/coal (w/w) and 4,08 $\mathrm{CS}_{2} / \mathrm{coal}(\mathrm{w} / \mathrm{w})$. Different tests such as acidity, kinetic and adsorption isotherms were carried out in order to assess the adsorption capacity of xanted and non-xanted coal. The sulfur groups presented in the xanted coal were identified by infrared spectroscopy(FTIR) and they were compared with non-xanted coal spectrum. The results show that the best lead removal in the solution (with a concentration of $100 \mathrm{mg} \mathrm{Pb}^{2+} / \mathrm{L}$ and $50 \mathrm{mg}$ of adsorbent) was obtained at $\mathrm{pH}$ 5.0 for both coal (xanted and non-xanted) with an adsorption percentage of $98 \%$. The kinetic studies indicate that the adsorption process can reach the equilibrium in about 120 minutes, following a pseudo-second order kinetics for both materials. The isotherms study indicates that the coal fits best to a Langmuir-type isotherm, however when the coal is xanted, it fits best to a Freundlich-type isotherm.

Key words: Coal, Langmuir isotherm, xanthates, industrial effluents.

\section{INTRODUCCIÓN}

La contaminación de los cuerpos de agua con plomo y otros metales pesados ha puesto en peligro la salud de seres humanos y animales, pues al no degradarse, los metales se pueden acumular en los tejidos vivos, causando diversas enfermedades y trastornos, por su efecto tóxico y cancerígeno (Wan \& Hanafiah, 2008). Actualmente, existen diferentes focos de contaminación por plomo en los cuerpos de agua de la bahía de Cartagena, a consecuencia de la actividad industrial que se lleva a cabo en la zona de mamonal, en especial, en las empresas del sector petroquímico, donde se hace necesario implementar sistemas de tratamiento adecuados. A fin de remover estos metales pesados de las aguas 
residuales, se han utilizado diversos métodos convencionales; sin embargo, estos métodos no son económicos ni eficaces, cuando la concentración de los metales se encuentra en un intervalo de concentración de 1 a $100 \mathrm{mgL}^{-1}$ (Torres et al. 2013).

Las unidades de intercambio iónico son sistemas de tratamiento de aguas residuales adecuados para la remoción de metales pesados, los cuales, utilizan un lecho relleno de material sólido, con propiedades de intercambio iónico, para la remoción de contaminantes. El material sólido, generalmente utilizado, consiste en una resina polimérica de origen sintético importada y fabricada en Estados Unidos.

La obtención de resinas de intercambio iónico a partir de recurso natural no renovable y abundante, como es el caso del carbón mineral, es una alternativa importante para el desarrollo de la investigación encaminada a la obtención de nuevo material de intercambio iónico, dada la importancia de su aplicación y de su valor comercial.

El carbón es un mineral que presenta en su composición química radicales carboxílicos y fenólicos, los cuales, lo hacen un sustrato ideal para la síntesis de xantatos, por su alto contenido de grupos funcionales hidroxilo, aumentando drásticamente la capacidad de intercambio de iones, por medio de la incorporación de grupos xantatos o sulfonación a la matriz carbonosa. Así lo demuestra el trabajo realizado por Orjuela et al. (2000), que obtuvo intercambiadores catiónicos de acidez fuerte, a partir de la sulfonación de cuatro carbones colombianos. Los grupos azufre, se caracterizan por tener una alta afinidad por metales pesados, pero una baja afinidad por metales ligeros. Los xantatos, se forman por reacción de un sustrato orgánico que contiene grupos hidroxilos con disulfuro de carbono, en condiciones básicas (Liang et al. 2009). Los carbones de medio rango presentan, en su estructura, un porcentaje importante de oxígeno, que lo hace ser importante para la incorporación de grupos xantatos, en la matriz carbonosa.

En estado natural, los carbones de bajo rango, como los lignitos y los sub-bituminosos, presentan alguna capacidad de intercambio, debido a la presencia de radicales carboxílicos y fenólicos, que son poco estables, de carácter débil, con facilidad de ionización y posterior desactivación en soluciones iónicas

La retención de metales pesados, a partir de la incorporación de grupos xantatos en materiales carbonosos ha sido materia de interés, destacando las investigaciones adelantadas por Haenel (1992), donde utilizó la xantación para aumentar el contenido de grupos funcionales, para la formación de complejos de metales en el carbón, a través de los electrones libre enlazantes del disulfuro de carbono $\mathrm{CS}_{2}$ (Khalir et al.
2012). En otro estudio, se prepararon resinas de intercambio iónico, a partir de xantación de residuos maderosos, obteniendo resultados importantes en la remoción de plomo en soluciones acuosas; sus resultados reflejaron el aumento en la remoción de plomo, entre el material xantado y sin xantizar (Nuñez et al. 2017). En esta investigación, se propone un proceso para el aprovechamiento de residuos agroindustriales, útiles en la obtención de resinas de intercambio iónico, a partir de residuos maderosos, de actividades forestales. Se prepara un adsorbente, a partir de la incorporación de grupos xantatos a la superficie del carbón y la evaluación de su factibilidad, para adsorber $\mathrm{Pb}^{2+}$ de soluciones acuosas, mediante estudios de adsorción, estudios cinéticos y de valores de $\mathrm{pH}$, favorables para la adsorción.

\section{MATERIALES Y MÉTODOS}

En esta investigación, se utilizó un diseño experimental de tipo factorial, a un solo nivel, para un solo tipo de carbón, ensayando tres valores de $\mathrm{pH}$ y realizando todas las experiencias por triplicado. La concentración de $\mathrm{Pb}^{2+}$ se determinó por espectrometría de absorción atómica de llama, en un equipo ICE 3000 Thermo Scientific, con corrección Zeeman. Las soluciones fueron preparadas aplicando una solución amortiguadora de acetato de sodio/ácido acético, ajustando la fuerza iónica con $\mathrm{NaCl}$. Todos los reactivos utilizados fueron de grado analítico.

Xantación del material adsorbente: Para realizar la xantación, se utilizó carbón sub-bituminoso de características térmicas favorables, bajo nivel de azufre, de cenizas y con bajas emisiones de óxidos de nitrógeno, procedente de la mina El Descanso, ubicada en el corregimiento de la Loma, del departamento del Cesar (Colombia).

Primeramente, el mineral fue sumergido en una solución de acetona de grado reactivo durante de siete días, para lograr su hinchamiento y transcurrido este periodo, se lavó varias veces con agua destilada, hasta obtener valores constantes en la conductividad del agua de lavado y se secó a $70^{\circ} \mathrm{C}$, por 24h. Posteriormente, se molió en un molino de bolas, para reducir su tamaño de partículas; se tamizó, para seleccionar un tamaño de partícula de $0,225 \mathrm{~mm}$; posteriormente, se lavó con $\mathrm{HCl} 0,1 \mathrm{~N}$ y, por último, con agua destilada, hasta $\mathrm{pH}$ neutro; luego, se secó a $70^{\circ} \mathrm{C}$ por $24 \mathrm{~h}$, para eliminar sustancias de color y posibles contaminantes. El producto obtenido se denominó C.

A continuación, se tomaron $25 \mathrm{~g}$ de carbón hinchado y del mismo tamaño de partícula y se introdujeron en un recipiente de polietileno de $2 \mathrm{~L}$, agitado mecánicamente. Se adicionaron $424 \mathrm{~mL}$ de una solución $4 \mathrm{M}$ de $\mathrm{NaOH}$, empleando una relación 16.6:1 NaOH/carbón p/p, agitando por tres horas; luego, $102 \mathrm{~mL}$ de $\mathrm{CS}_{2}$ puro, con una relación 4,08:1 CS/ 
carbón p/p, agitando por cuatro horas; se dejó la mezcla en reposo por 16 horas y, posteriormente, se realizó un filtrado; se lavó varias veces con agua desionizada y se secó, a una temperatura de $70^{\circ} \mathrm{C}$, de acuerdo con la metodología de Saswati \& Vinod (2006). Este producto se denominó CS.

Experimentos de adsorción: El efecto del $\mathrm{pH}$ sobre la adsorción de $\mathrm{Pb}^{2+}$ se examinó a partir de la mezcla de $50 \mathrm{mg}$ de CS y C, con $10 \mathrm{~mL}$ de solución de $100 \mathrm{ppm}$ de $\mathrm{Pb}^{+}$, en un beaker de $100 \mathrm{~mL}$, a $25^{\circ} \mathrm{C}$. Los análisis, se realizaron a valores de $\mathrm{pH}$, comprendidos entre 3,0 y 5,0 ajustando mediante la adición de solución $0,1 \mathrm{M}$, de $\mathrm{HNO}_{3}$ y $0,1 \mathrm{M}$, de $\mathrm{NaOH}$. Después de tres horas de agitación, las soluciones se filtraron y se determinó la concentración del filtrado. Para el estudio cinético de $\mathrm{Pb}^{2+}$, se mezclaron $500 \mathrm{mg}$ de adsorbente, con $100 \mathrm{~mL}$ de solución, a una concentración de 100ppm de $\mathrm{Pb}^{2+}$, en un Erlenmeyer de $250 \mathrm{~mL}$, a un valor de $\mathrm{pH}=5,0$ durante $2,2 \mathrm{~h}, \mathrm{a} 25^{\circ} \mathrm{C}$. Posteriormente, se extrajeron muestras a diferentes intervalos de tiempo y se sometieron a un proceso de filtraron, para luego determinar su concentración. Los experimentos de adsorción para $\mathrm{Pb}^{2+}$ fueron realizados en un beaker de $100 \mathrm{~mL}$, mezclando $50 \mathrm{mg}$ de adsorbente, con $10 \mathrm{~mL}$ de solución de $\mathrm{Pb}^{2+}$ de 25, 50,100, 200 o 400ppm, a un $\mathrm{pH}=5$, durante dos horas, a $25^{\circ} \mathrm{C}$. La cantidad de metal adsorbido se determinó usando la ecuación 1 (Nuñez et al. 2017):

$$
q_{e}=\frac{\mathrm{C}_{0} \mathrm{~V}_{0}-\mathrm{C}_{\mathrm{e}} \mathrm{V}_{0}}{\mathrm{~m}}
$$

Donde: Cantidad de metal adsorbido; $C_{0}$ es la concentración inicial de $\mathrm{Pb}^{2+}$ en $\mathrm{mgL}^{-1}$; $\mathrm{V}_{0}$ el volumen inicial de la solución en $\mathrm{L} ; \mathrm{C}_{\text {e, }}$ la concentración en el equilibrio de $\mathrm{Pb}^{2+}$ en $\mathrm{mgL}^{-1}$ y $\mathrm{m}$ es la masa del adsorbente.

Caracterización de los adsorbentes: Los espectros infrarrojos de $\mathrm{C}$ y CS, se obtuvieron mezclando y macerando $1 \mathrm{mg}$ de muestra con $100 \mathrm{mg}$ de $\mathrm{KBr}$, comprimiendo, para obtener una pastilla, que se analizó en un equipo de FTIR
(SHIMADZU-8400), en el rango de $4000-400 \mathrm{~cm}^{-1}$, para identificar los grupos funcionales que intervienen en la adsorción de metales pesados.

Análisis estadísticos: Los datos, se analizaron mediante ANOVA (una vía), para determinar diferencias estadísticamente significativas $(P<0,05)$ entre las muestras. Se utilizó el software SPSS (versión 17.0 para Windows). Todos los ensayos se realizaron por triplicado.

\section{RESULTADOS Y DISCUSIÓN}

El análisis próximo y último del carbón mineral, se realizó según las normas ASTM; estos resultados se aprecian en la tabla 1. De acuerdo con los resultados del análisis próximo y último se puede afirmar, que el carbón procedente de la mina El Descanso es de tipo subbituminoso, cuya macro estructura presenta grupos funcionales y compuestos alifáticos, que actúan como fase móvil, que lo hacen importante para darle funcionabilidad a la matriz carbonosa, mediante la reacción de xantación, que permite la incorporación de los grupos xantatos.

Efecto del pH: En la figura 1, se observa que el pH es un factor que afecta la adsorción de metales pesados en solución acuosa. El porcentaje de plomo adsorbido se determinó mediante la ecuación 2:

$$
\mathrm{Q}=(\mathrm{Ci}-\mathrm{Cf}) * \mathrm{~V} / \mathrm{m}
$$

Donde: $\mathrm{Q}$ es la capacidad de adsorción (mg de metal/g de adsorbente); V es el volumen de la solución $(\mathrm{mL})$; $\mathrm{Ci}$ es la concentración inicial de metal en solución (mg/L); Cf es la concentración en el equilibrio de la solución ( $\mathrm{mg} / \mathrm{L}$ ) y m es la cantidad de adsorbente seco.

A valor de $\mathrm{pH}$ bajo se observa una pobre adsorción de $\mathrm{Pb}^{2+}$, como resultado de la fuerte repulsión electrostática de los

Tabla 1. Análisis próximo y elemental del carbón sin xantar.

\begin{tabular}{|c|c|c|c|}
\hline \multicolumn{2}{|c|}{ Análisis próximo del carbón sin xantar (\%) } & \multicolumn{2}{c|}{ Análisis elemental del carbón sin xantar (\%) } \\
\hline Humedad & $8,10 \pm 0,006$ & Azufre & $0,230 \pm 0,011$ \\
\hline Material volátil & $35,1 \pm 0,260$ & Carbono & $58,30 \pm 0,620$ \\
\hline Cenizas & $3,40 \pm 0,025$ & Nitrógeno & $1,410 \pm 0,070$ \\
\hline Carbón fijo & $53,4 \pm 0,260$ & Hidrógeno & $3,680 \pm 0,090$ \\
\hline Poder calorífico & $1,50 \pm 0,025$ & Oxígeno & $36,31 \pm 0,602$ \\
\hline
\end{tabular}

Los valores representan la media de tres determinaciones. 


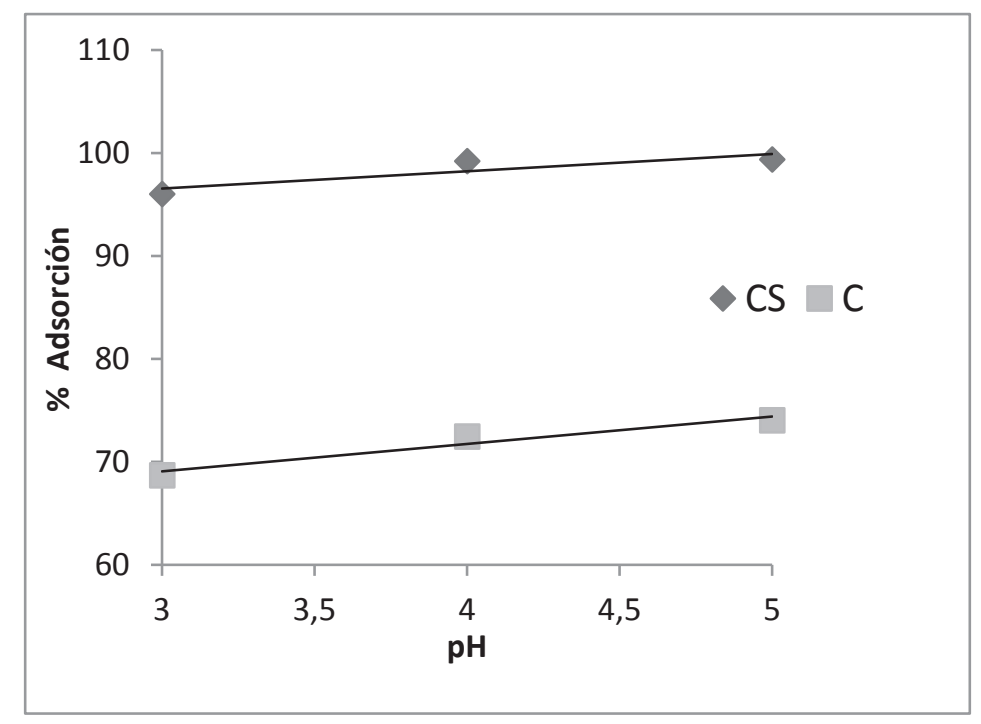

Figura 1. Efecto del pH en la adsorción de $\mathrm{Pb}^{2+}$ con $\mathrm{C}$ y CS.

iones $\mathrm{H}^{+}$en la superficie del carbón con los iones metálicos, los cuales, impiden que estos interactúen con el adsorbente (Saswati \& Vinod, 2006). Al aumentar el pH del medio, la fuerza de repulsión se hace débil, favoreciendo la difusión de los iones de $\mathrm{Pb}^{2+}$ hacia la superficie del adsorbente, donde aumenta la adsorción (Jiang et al. 2009). De la figura 1 , se infiere que la máxima adsorción de C y CS se obtiene a $\mathrm{pH}=5,0$, con porcentajes de 74 y $99 \%$ de soluciones de 100 ppm de $\mathrm{Pb}^{2+}$ y dosis de $50 \mathrm{mg}$ de adsorbente/10mL, respectivamente. La principal ventaja de CS sobre C es que la adsorción de $\mathrm{Pb}^{2+}$ es mayor para CS a todos los valores de $\mathrm{pH}$. Lo anterior concuerda con lo reportado por Torres et al. (2013), al evaluar la adsorción de $\mathrm{Pb}^{2+}$ en cáscara de pimienta y cáscara de pimienta xantada; observaron que a $\mathrm{pH}$ bajos la adsorción de la cáscara de pimienta es pobre, como resultado de la fuerte repulsión electrostática, mientras que la adsorción de $\mathrm{Pb}^{2+}$ es grande para la cáscara de pimienta xantada, en todos los intervalos de $\mathrm{pH}$; resultados similares fueron obtenidos por Taty-Costodes et al. (2003), Liang et al. (2009) y Liang et al. (2011).

Cinética de adsorción: Una de las propiedades más importantes de los adsorbentes es la rapidez con la que el adsorbato es retenido y alcanza el equilibrio. El modelo matemático que describe la cantidad de $\mathrm{Pb}^{2+}$ adsorbido de la solución por el adsorbente, en un intervalo de tiempo, están dadas por la ecuación 3 (Núñez et al. 2017):

$\left(\frac{\mathrm{mg} \mathrm{Pb}^{2+}}{\mathrm{g}}\right)_{\mathrm{n}}=\frac{\left[(\mathrm{CV})_{\mathrm{n}-1}-(\mathrm{CV})_{\mathrm{n}}\right]}{\mathrm{g}}+\left(\frac{\mathrm{mg} \mathrm{Pb}^{2+}}{\mathrm{g}}\right)_{\mathrm{n}-1}$

Donde: $\mathrm{C}$ es la concentración remanente de $\mathrm{Pb}^{2+}$ en $\mathrm{mgL}^{-1}$ en un tiempo $t$; $V$ es el volumen de la solución remanente en un tiempo $t$ en $\mathrm{L}$ y $\mathrm{n}$ es el número de datos que han sido tomados.

La gráfica de $\mathrm{Pb}^{2+}$ adsorbido como función del tiempo, permite identificar el momento en que se alcanza el equilibrio y la correspondiente capacidad de adsorción $q_{e}$ (Silgado et al. 2014). Para evaluar la tasa de adsorción $\mathrm{Pb}^{2+}$ en $\mathrm{C}$ y CS, se consideraron dos modelos cinéticos (pseudo primer y pseudo segundo orden.

El modelo cinético de pseudo-primer orden fue propuesto por Lagergren y se expresa mediante la ecuación 3 (Núñez et al. 2017), donde: qe y qt son las capacidades de adsorción $\left(\mathrm{mgg}^{-1}\right)$ en el equilibrio y en el tiempo $\mathrm{t}$, respectivamente, $\mathrm{y} \mathrm{k}_{1}$ es la constante de velocidad del modelo de pseudo-primer orden $\left(\mathrm{min}^{-1}\right)$. Los valores de $\mathrm{k}_{1}$ se obtienen a partir de la intersección y la pendiente de la gráfica de $\log \left(q_{e}-q_{t}\right)$ Vs t:

$$
\log \left(q_{e}-q_{t}\right)=\log q_{e}-\frac{k_{1}}{2.303} t
$$

El modelo cinético de pseudo-segundo orden está dado por la ecuación 5, donde: $q_{\text {e }}$ y $q_{t}$ se definen como en el modelo de pseudo-primer orden y $\mathrm{k} 2$ es la constante de velocidad del modelo de pseudo-segundo orden $\left(\mathrm{gmg}^{-1} \mathrm{~min}^{-1}\right.$ ) (Torres et al. 2013). Los valores de $\mathrm{k}_{2}$ y qe se pueden obtener a partir de la intersección y la pendiente de la gráfica de $\mathrm{t} / \mathrm{q}_{\mathrm{t}} \mathrm{Vs} \mathrm{t}$ (Nuñez et al. 2017; Silgado et al. 2014):

$$
\frac{\mathrm{t}}{\mathrm{q}_{\mathrm{t}}}=\frac{1}{\mathrm{k}_{2} \mathrm{q}_{\mathrm{e}}^{2}}+\frac{1}{\mathrm{q}_{\mathrm{e}}} \mathrm{t}
$$

Este modelo también supone que la velocidad de adsorción inicial ho $\left(\mathrm{mgg}^{-1} \mathrm{~min}^{-1}\right)$ es proporcional al cuadrado del nú- 
mero de sitios restantes de superficie libre y viene definida, como se expresa por la ecuación 6 (Núñez et al. 2017):

$$
\mathrm{h}_{0}=\mathrm{k}_{2} \mathrm{q}_{\mathrm{e}}^{2}
$$

Donde: qe y k2 son la capacidad de adsorción $\left(\mathrm{mgg}^{-1}\right)$ y la constante de velocidad del modelo de pseudo-segundo orden $\left(\mathrm{gmg}^{-1} \mathrm{~min}^{-1}\right)$. Los resultados obtenidos para el qe de cada modelo, las respectivas constantes cinéticas y el coeficiente de regresión $\mathrm{R}^{2}$, se describen en la tabla 2 .
Se observa que para el modelo cinético de pseudo-primer orden, los valores teóricos de qe para C y CS difieren respecto a los $\mathrm{q}_{\mathrm{e}}$ experimentales, esto se evidencia en el coeficiente de regresión de 0,919 y 0,901, para C y CS, respectivamente. En cuanto al modelo de pseudo-segundo orden, los valores de qe experimental concuerdan con los qe teóricos para ambos adsorbentes, con un coeficiente de regresión de 1 , para C y CS; por lo tanto, el mecanismo de adsorción de los iones de $\mathrm{Pb}^{2+}$ en $\mathrm{C}$ y CS están representados por el modelo cinético de pseudo-segundo orden, de acuerdo con lo repor-

Tabla 2. Parámetros cinéticos para C y CS. Parámetros isotérmicos de Langmuir y Freundlich para la adsorción de $\mathrm{Pb}^{2+}$ en C y CS.

\begin{tabular}{|c|c|c|c|c|c|c|c|c|c|c|}
\hline \multicolumn{11}{|c|}{ Modelo cinético } \\
\hline \multirow[b]{2}{*}{ Adsorbente } & \multicolumn{5}{|c|}{ Pseudo primer orden } & \multicolumn{5}{|c|}{ Pseudo segundo orden } \\
\hline & $\begin{array}{c}\mathrm{qe}_{\mathrm{e}} \\
\text { (exp) }\end{array}$ & $\begin{array}{l}\text { qe modelo } \\
\left(\mathrm{mg} \mathrm{g}^{-1}\right)\end{array}$ & & \multicolumn{2}{|c|}{$\mathrm{R}^{2}$} & $\begin{array}{l}\text { qe modelo } \\
\left(\mathrm{mg} \mathrm{g}^{-1}\right)\end{array}$ & \multicolumn{2}{|c|}{$\underset{\left(\mathrm{g} \mathrm{mg}_{1}^{-1}\right)}{\mathrm{K}_{2}} \min ^{-}$} & $\mathrm{R}^{2}$ & $\begin{array}{c}\mathrm{H}_{0} \\
\left(\mathrm{mg} \mathrm{g}^{-1}\right)\end{array}$ \\
\hline C & 16,752 & 15,98 & & \multicolumn{2}{|c|}{0,919} & 17,311 & \multicolumn{2}{|c|}{0,018} & 1 & 5,980 \\
\hline \multirow[t]{2}{*}{ CS } & 19,816 & 19,70 & & \multicolumn{2}{|c|}{0,901} & 19,594 & \multicolumn{2}{|c|}{0,036} & 1 & 14,18 \\
\hline & \multicolumn{10}{|c|}{ Isotermas de adsorción } \\
\hline \multirow[b]{2}{*}{ Adsorbente } & \multicolumn{5}{|c|}{ Langmuir } & \multicolumn{5}{|c|}{ Freundlich } \\
\hline & \multicolumn{2}{|c|}{$\begin{array}{c}\mathrm{q}_{\mathrm{m}} \\
\mathrm{mg} \mathrm{g}^{-1}\end{array}$} & & $\mathrm{R}^{2}$ & RL & \multicolumn{2}{|c|}{$\mathrm{K}_{F}, \mathrm{~L} \mathrm{~g}^{-1}$} & \multicolumn{2}{|c|}{$\mathrm{N}$} & $\mathrm{R}^{2}$ \\
\hline C & \multirow{2}{*}{\multicolumn{2}{|c|}{10,44}} & & 0,971 & 0,035 & \multicolumn{2}{|c|}{1.042} & \multicolumn{2}{|c|}{0,394} & 0,914 \\
\hline CS & & 60,10 & & 0,530 & 0,320 & & 39 & & & 0,981 \\
\hline
\end{tabular}

tado por Torres et al. (2013), Liang et al. (2011) y Liang et al. (2009), quienes evaluaron la adsorción de plomo en cáscara de pimienta y de naranja; esto indica que la adsorción química es el mecanismo de control de la velocidad. En cuanto a la velocidad inicial, Ho es mayor para el carbón xantado, lo que confirma que la xantación aumenta la afinidad del bioadsorbente hacia los metales.

Isotermas de adsorción: Se evaluaron utilizando el modelo de Langmuir y Freundlich. La isoterma de Langmuir viene dada por la ecuación 6 , donde: qe es la cantidad de material adsorbido por una cantidad específica de adsorbente $\left(\mathrm{mgg}^{-1}\right) ; \mathrm{C}_{\mathrm{e}}$ es la concentración de equilibrio $\left(\mathrm{mgL}^{-1}\right) ; \mathrm{qm}_{\mathrm{m}}$ es la cantidad de metal o contaminante necesario para formar una monocapa en la superficie $\left(\mathrm{mgg}^{-1}\right)$ y expresa la capacidad máxima de adsorción del adsorbente, mientras que b es la constante de Langmuir $\left(\mathrm{Lmg}^{-1}\right.$ ) (Núñez et al. 2017; Silgadoet al. 2014; Śćiban et al. 2006):
$\frac{\mathrm{C}_{\mathrm{e}}}{\mathrm{q}_{\mathrm{e}}}=\frac{1}{\mathrm{q}_{\mathrm{m}} \mathrm{b}}+\frac{\mathrm{C}_{\mathrm{e}}}{\mathrm{q}_{\mathrm{m}}}$

Las características esenciales de la isoterma de Langmuir, se pueden expresar en términos de una dimensión constante, conocido como el factor de separación RL, que está dado por la ecuación 8:

$$
\mathrm{RL}=\frac{1}{1+\mathrm{bCo}}
$$

Donde: $\mathrm{b}$ es la constante de Langmuir y $\mathrm{C}_{\mathrm{o}}$ es la concentración inicial de sorbato en la solución, descritas anteriormente. El factor de separación RL indica la forma de la isoterma, si la adsorción es favorable o no, de acuerdo con los siguientes criterios: si $R L>1$, es desfavorable; $R L=1$, lineal; $0<R L<1$, favorable y $\mathrm{RL}=0$, la adsorción es irreversible (Núñez et al. 2017; Memon et al. 2007). A diferencia de la isoterma de Langmuir, que supone una superficie homogénea, la isoterma de Freundlich considera una superficie heterogénea. La isoterma de Freundlich, se utiliza a presiones bajas y está 
representada por la ecuación 9, donde: qe es la cantidad de material adsorbido por una cantidad específica de adsorben-

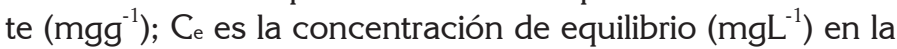
fase líquida; $\mathrm{K}_{\mathrm{F}}$ es la constante de Freundlich y $\mathrm{n}$ el factor de heterogeneidad, el cual, depende de la sustancia (Núñez et al. 2017; Liang et al. 2009):

$$
\log \mathrm{qe}=\log \mathrm{KF}+\frac{1}{\mathrm{n}} \log \mathrm{Ce}
$$

Las figuras $2 \mathrm{a}$ y $2 \mathrm{~b}$ ilustran los resultados obtenidos para $\mathrm{C}$ y CS.

Las isotermas de la figura 2a muestran que la capacidad de adsorción en el equilibrio $C_{e}$ incrementa, como una función de la concentración qe en la solución, mientras que la figura $2 \mathrm{~b}$ confirma que la adsorción de $\mathrm{Pb}^{2+}$, mediante $\mathrm{C}$ y CS es favorable, pues el valor de RL para todas las concentraciones de metal, se encuentran en el intervalo de $0-1$. Según Kumar et al. (2000) esto quiere decir que la adsorción es más favorable a concentraciones más altas.

En la tabla 2, se observan los parámetros cinéticos para $\mathrm{C}$ y CS y los parámetros isotérmicos de Langmuir y Freundlich, para la adsorción de $\mathrm{Pb}^{2+}$ en $\mathrm{C}$ y CS, los cuales, se determinaron por ajuste de mínimos cuadrados. Para determinar cuál de los dos modelos de isotermas se ajusta mejor a la adsorción de $\mathrm{Pb}^{2+}$, se evaluaron los coeficientes de correlación, una medida que permite conocer cómo se ajustan los valores pronosticados con los valores experimentales (Pillai et al.
2013; Varga et al. 2013; Putra et al. 2014). Los resultados presentan coeficientes de correlación con respecto a la adsorción de $\mathrm{Pb}^{2+}$ en $\mathrm{C}$, n de 0,971 y 0,914 , respectivamente, para un nivel de significancia del 0,05, indicando que el modelo que mejor se ajusta es el de Langmuir, en comparación con el modelo Freunlich.

La adsorción de $\mathrm{Pb}^{2+}$ se da sobre una superficie de adsorbente homogénea, que forma una monocapa, donde cada sitio de adsorción puede adherir una sola molécula de adsorbato y tienen la misma energía de adsorción. Para el CS, se observa que los coeficientes de correlación fueron de 0,53 y 0,981, para los modelos de Langmuir y Freundlich, respectivamente, indicando que la adsorción sigue la isoterma de Freundlich; esto significa, que la remoción de $\mathrm{Pb}^{2+}$ ocurre, debido a la distribución de energía de los sitios activos para adsorción y la ausencia de monocapas. Lo anterior concuerda con lo investigado por Liang et al. (2009), quienes estudiaron la adsorción de $\mathrm{Pb}^{2+}$ en cáscara de naranja y en cáscara de naranja xantada y concluyeron que la adsorción de $\mathrm{Pb}^{2+}$ en la cáscara de naranja sigue una isoterma de tipo Freundlich, pero una vez xantada, sigue una isoterma tipo Langmuir. Por otra parte, Torres et al. (2013) establecieron que la adsorción de $\mathrm{Pb}^{2+}$, a partir de cáscara de pimienta xantada y no tratada, sigue una isoterma de tipo Freundlich, lo que quiere decir que el tipo de isoterma de adsorción depende de la naturaleza del bioadsorbente utilizado (Bsoul et al. 2014).

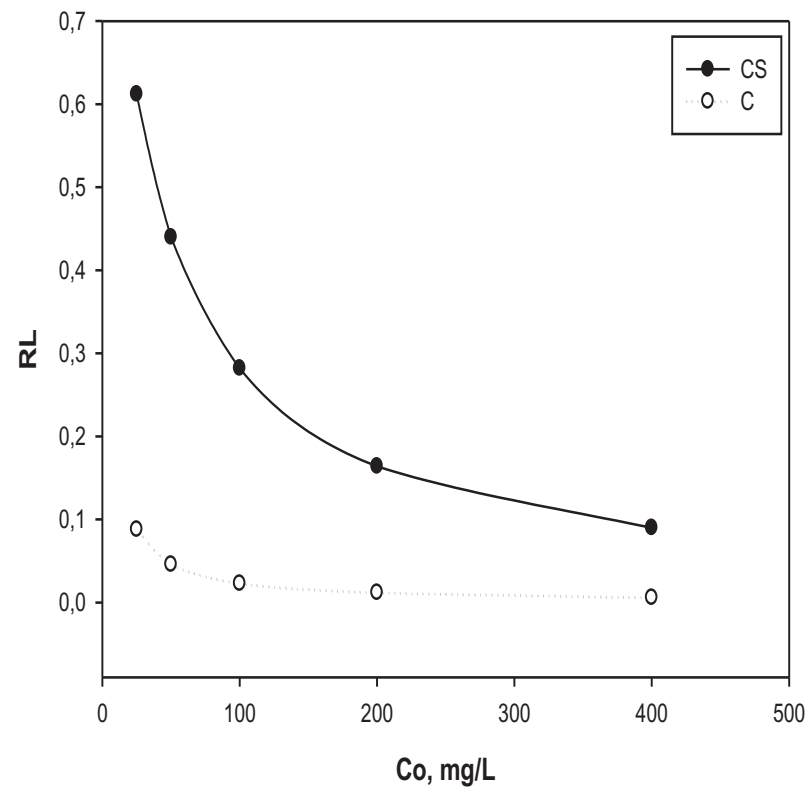

Figura 2. A) Isoterma de Langmuir y Freundlich para la adsorción de $\mathrm{Pb}^{2}+$ en $\mathrm{C}$ y CS; B) Factor de separación en la adsorción de $\mathrm{Pb}^{2+}$ en $\mathrm{C}$ y CS. 
Espectroscopia infrarroja: Los espectros FTIR de C y CS se muestran en las figuras 3 y 4 . En el espectro de $C$, los picos anchos e intensos de absorción alrededor de $3442 \mathrm{~cm}^{-1}$, corresponden a vibraciones de tensión del enlace $\mathrm{O}-\mathrm{H}$, tales como alcoholes y fenoles, presentes en el carbón. Los picos a 2920 y $2851 \mathrm{~cm}^{-1}$, se atribuyen a la tensión del enlace $\mathrm{C}$-H de los grupos alcanos, debida a los grupos $-\mathrm{OCH}_{3}$ y $-\mathrm{CH}_{2} \mathrm{OH}$, presentes en la estructura del carbón, como la lignina (Liang et al. 2011). El pico observado a $1696 \mathrm{~cm}^{-1}$ es el resultado de la vibración de tensión del enlace $\mathrm{C}=\mathrm{O}$, debido a los grupos carbonilo de la lignina. Los picos que aparecen a 1648, 1616, 1560, 1542, 1510, 1456, 1431 y $1423 \mathrm{~cm}^{-1}$, se deben a vibraciones de tensión de los enlaces $\mathrm{C}=\mathrm{C}$ de los grupos aromáticos de la lignina. Los picos a 1313, 1338, 1190, 1167 y $1114 \mathrm{~cm}^{-1}$, se asignan a la torsión o deformación O-H y vibraciones de tensión de C-O, de alcoholes pri- marios y secundarios. Algunos cambios se observaron en el espectro de CS con respecto al de C.

La presencia de los grupos xantatos en el CS, se identifican por la aparición de nuevos picos, a 610 y 1033,88cm ${ }^{-1}$, que corresponden a los enlaces $\mathrm{C}-\mathrm{S} \gamma \mathrm{C}=\mathrm{S}$; concuerda con lo reportado por Pillai et al. (2013), quien identificó la incorporación de grupos azufre por la aparición de picos, a 538, 1020 y $1151,5 \mathrm{~cm}^{-1}$, que corresponden a las vibraciones C-S, $\mathrm{C}=\mathrm{S}$ y S-C-S. Por otro lado, Panda et al. (2008) identificaron la presencia grupos xantatos en cáscara de Lathyrus mativus por los picos aparecidos, a 661,5, 1080,5, 1163 y 1200 a $1250 \mathrm{~cm}^{-1}$. Mustafa et al. (2004) sostienen que las bandas de absorción característicos de los grupos xantatos, se encuentran en la región de $800-1200 \mathrm{~cm}^{-1}$ (Núñez et al. 2016).

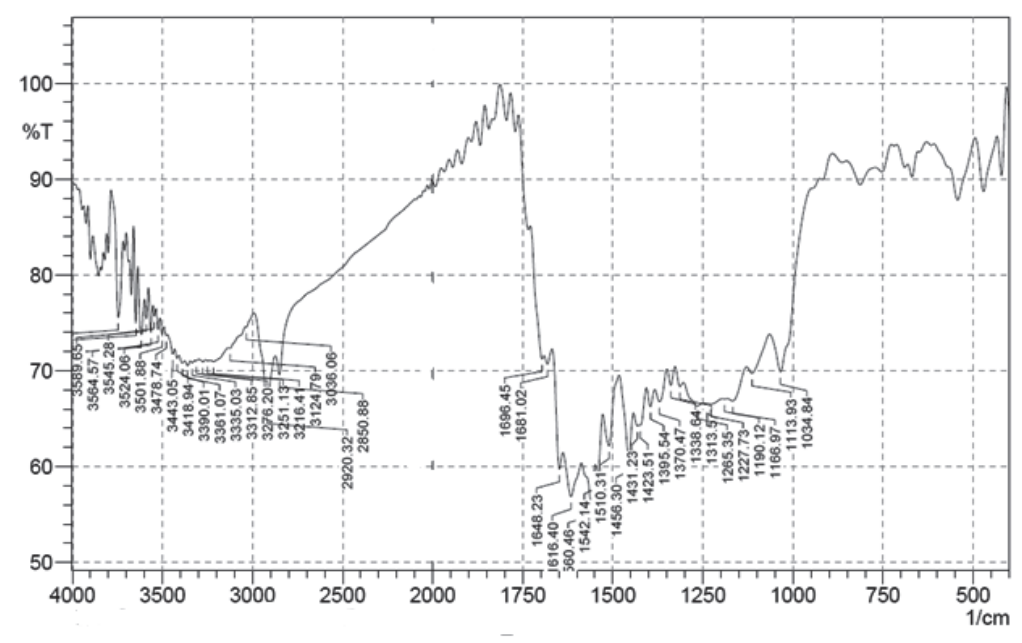

Figura 3. Espectro FTIR de C.

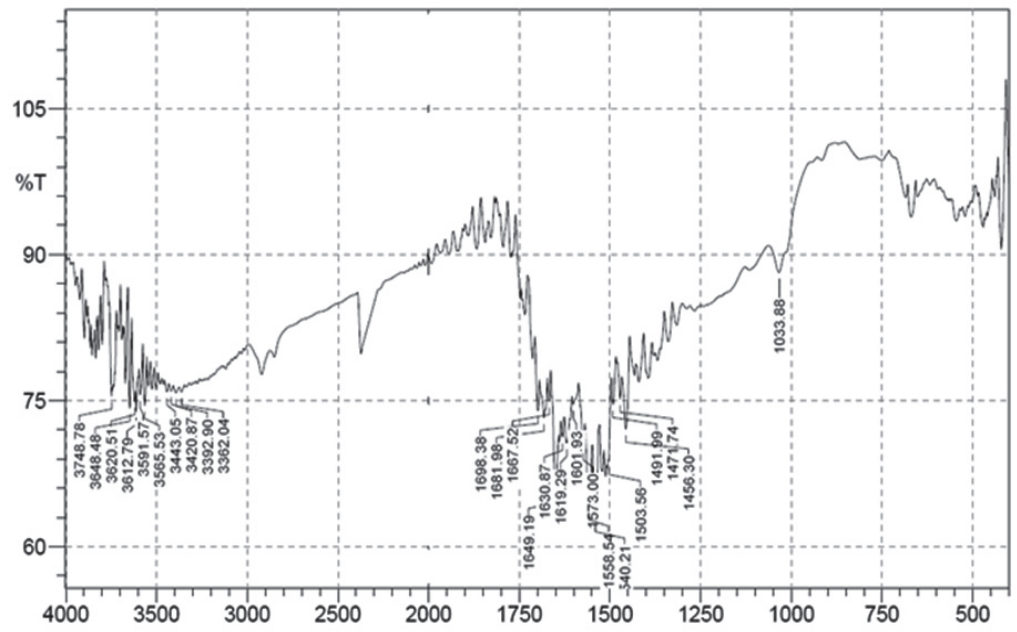

Figura 4. Espectro FTIR de CS. 
El carbón xantado presenta un gran potencial como un adsorbente eficiente y útil para la adsorción de $\mathrm{Pb}^{2+}$, pues su capacidad de adsorción es de $60 \mathrm{mg} \mathrm{Pb}^{2+}$ por gramos de adsorbente, ajustándose la adsorción a la isoterma de Freundlich, mientras que el carbón sin xantar, se ajusta a la isoterma de Langmuir. El pH del medio es un factor que afecta la adsorción de $\mathrm{Pb}^{2+}$ en solución acuosa, siendo el $\mathrm{pH}=5,0$ el punto donde se observó la mayor remoción, con porcentajes de adsorción de 74 y 99\%, para el carbón y carbón xantado, respectivamente.

El análisis cinético mostró que el equilibrio de adsorción es alcanzado en, aproximadamente, $120 \mathrm{~min}$, siguiendo una cinética de pseudo-segundo orden, para ambos materiales. Los cambios observados en los espectros infrarrojos muestran que, efectivamente, se realizó la incorporación de los grupos xantatos en la estructura química del carbón.

Agradecimientos: A todos los miembros de los grupos de investigación Carboquímica y GIBAE de la Universidad de Cartagena. Conflicto de intereses: El manuscrito fue preparado y revisado con la participación de todos los autores, quienes declaramos que no existe conflicto de intereses que ponga en riesgo la validez de los resultados presentados.

\section{BIBLIOGRAFÍA}

1. BSOUL, A.; ZEATOUN, L.; ABDELHAY, A.; CHIHA, M. 2014. Adsorption of copper ions from water by different types of natural seed materials. Desalination Water Treat. (United Kingdom). 52:5876-5882.

2. HAENEL, M. 1992. Recent progress in coal structure research. Fuel. (Netherlands). 71:1211-1223.

3. JIANG, Y.; PANG, H.; LIAO, B. 2009. Removal of copper (II) ions from aqueous solution by modified bagasse. J. Hazard. Mater. (Netherlands). 164:1-9.

4. KHALIR, W.; HANAFIAH, M.; SOAD, S.; NGAH, W.; MAJID, Z. 2012. Batch column and thermodynamic of $\mathrm{Pb}$ (II) adsorption on xanthated rubber (Hevea brasiliensis) leaf powder. J. Applied Sciences. (Pakistan). 12:1142-1147.

5. KUMAR, A.; RAO, N.N.; KAUL, S.N. 2000. Alkali-treated straw and insoluble straw xanthate as low cost adsorbents for heavy metal removal - preparation, characterization and application. Bioresource Tecnol. (Netherlands). 71:133-142.

6. LIANG, S.; GUO, X.; FENG, N.; TIAN, Q. 2009. Application of orange peelxanthate for the adsorption of $\mathrm{Pb}^{2+}$ from aqueous solutions. J. Hazard. Mater. (Netherlands). 170: 425-429.

7. LIANG, S.; GUO, X.; TIAN, Q. 2011. Adsorption of $\mathrm{Pb}^{2+}$ and $\mathrm{Zn}^{2+}$ from aqueous solutions by sulfured orange peel. Desalination. (Netherlands). 275:212-216.

8. MEMON, S.Q.; SHAH, N.W.; KHUHAWAR, M.Y.; BHANGER, M.I. 2007. Sawdust-A green and economical sorbent for the removal of cadmium (II) ions. J. Hazard. Mater. 139(1):116-121.

9. MUSTAFA, S.; HAMID, A.; NAEEM, A. 2004. Xanthate adsorption studies on chalcopyrite ore. Int. J. of Min. Process. (Netherlands). 74:317-325.

10. NUÑEZ, J.; COLPAS, F.; TARÓN, A. 2017. Aprovechamiento de residuos maderosos para la obtención de resinas de intercambio iónico. Rev. Temas Agrarios. (Colombia). 22(1):53-60.

11. NUÑEZ, J.; COLPAS, F.; FERNANDEZ, R. 2016. Xantação de resíduos de sawdust para adsorção de íons de chumbo a partir de soluções aquosas. Periódico Tche Quimica. (Brasil). 14(27):131-138.

12. ORJUELA, A.; CIVETTA, N.; RIVERA, J.; BOYACÁ, A.; DÍAZ, J. 2000. Preparación de intercambiadores catiónicos a partir de carbón. Rev Col. de Química. 29(2):53-59.

13. PANDA, G.K.; DAS, S.K.; GUHA, A.K. 2008. Niquely biosorption of cadmium in the shell functionalized Lathyrus sativus. Colloid Surf. (Netherlands). 62:173179.

14. PILLAI, S.; ELDHO ABRAHAM, D.; GIRIJA, N.; GEETHA, P.; JACOB, L.; KOSHY. M. 2013. Biosorption of Cd(II) from aqueous solution using xanthated nano banana cellulose: Equilibrium and kinetic studies. Ecotoxicol. Environm. Safety. (United States). 98:352-360.

15. PUTRA, W.; KAMARI, A.; YUSOFF, S.; ISHAK, C.; MOHAMED, A. 2014. Biosorption of $\mathrm{Cu}(\mathrm{II}), \mathrm{Pb}$ (II) and $\mathrm{Zn}$ (II) ions from aqueous solutions using selected waste materials: adsorption and characterisation studies. J. Encapsulation Adsorpt. Sci. (China). 4(1):25-35.

16. SASWATI, C.; VINOD, T. 2006. Role of various parameters in synthesis of insoluble agrobased xanthates for removal of copper from wastewater. Bioresour. Tecnol. (Netherlands). 97:2407-2413. 
17. SILGADO, K.J.; MARRUGO, G.D.; PUELLO, J. 2014. Adsorption of cromium (VI) by activated carbon produced from oil palm endocarp. Chem. Eng. Trans. (Italy). 37:721-726.

18. ŠĆIBAN, M.; KLAŠNJA, M.; ŠKRBIĆ, B. 2006. Modified softwood sawdust as adsorbent of heavy metal ions from water. J. Hazard. Mater. 136: 266-271.

19. TATY-COSTODES, V.C.; FAUDUET, H.; PORTE, C.; DELACROIX, A. 2003. Elimination of Cd (II) and Pb (II) ions starting from aqueous solutions by adsorption on sawdust. J. Hazard. Mater. 105:21-142.

20. TORRES, T.; ROA, G.; FAL, C.; BARRERA, C.; UREÑA, F.; PAVÓN, T.H. 2013. Improving lead sorption through chemical modification of de-oiled allspice husk by xanthate. Fuel. (Netherlands). 110:4-11.

21. VARGA, M.; TAKÁCS, M.; ZÁRAY, G.; VARGA, I. 2013. Comparative study of sorption kinetics and equilibrium of chromium (VI) on charcoals prepared from different low-cost materials. Microchem J. (Netherlands). 107:25-30.

22. WAN NGAH, W.; HANAFIAH, M. 2008. Removal of heavy metal ions from wastewater by chemically modified plant wastes as adsorbents: A review, Bioresour. Tecnol. (Netherlands). 99:3935-3948.

Recibido: Mayo 17 de 2017

Aceptado: Octubre 2 de 2017

Cómo citar:

Colpas Castillo, F.; Tarón Dunoyer, A.; Vásquez, Y. 2017. Capacidad de adsorción de plomo con intercambiadores catiónicos de carbón xantado. Rev. U.D.C.A Act. \& Div. Cient. 20(2): 425-433. 